\title{
REVIEW OF THE SYSTEM \\ OF ASTRONOMICAL CONSTANTS
}

\author{
By G. A. WILKINS,
}

H. M. Nautical Almanac Office, England.

RÉsumé. - L'auteur discute le but d'un système de constantes astronomiques ainsi que les principes de la construction d'un tel système. Il étudie les relations entre les constantes et propose une définition de chacune d'entre elles, faisant une nette distinction entre les constantes fondamentales et celles qui en sont dérivées. Le système obtenu diffère en partie du système actuel. L'auteur discute les étapes successives de l'adoption d'un nouveau système de constantes.

Abstract. - The purpose and principles of construction of a system of astronomical constants are described. The relationships between the constants are considered; all constants are defined precisely and a clear distinction between fundamental and derived constants is made. The system thus developed differs in some respects from the current system. The practical steps required to implement the formal adoption of such a new system are discussed.

Zusammenfassung. - Der Zweck und die Grundsätze der Aufstellung eines Systems astronomischer Konstanten werden beschrieben. Die Beziehungen zwischen den Konstanten werden untersucht; alle Konstanten werden genau definiert und dabei eine klare Unterscheidung zwischen fundamentalen und abgeleiteten Konstanten gemacht. Das so entwickelte System ist in mancher Hinsicht von dem jetzt gebräuchlichen System verschieden. Die praktischen Schritte, die zur formalen Annahme eines derartigen neuen Systems führen, werden diskutiert.

Резюме. - Автор обсуждает цель систем астрономических постоянных и принципы их построения. Он изучает соотношения между постоянными, и предлагает определения для каждой из них, делая решительное различие между фундаментальными постоянными и теми, которые от них происходят. Полученная система отчасти отличается от ныне принятой. Автор обсуждает последовательные ступени для принятия новой системы постоянных.

1. Intrbduction. - 1. I. Purpose of THE Review. - 1. I. I. The principal purpose of this review of the system of astronomical constants is to summarise the relationships between the constants, so that the 
numerical consequences of changing any of the fundamental constants may be readily evaluated. In specifying the relationships between the constants I have thereby constructed a self-consistent system in which there is a clear distinction between fundamental and derived constants. The principles that I have followed are described in the remaining sections of this Introduction. This new system differs in several respects from the current system, which is not clearly defined and for which the adopted values are not consistent with each other. I have made much use of the recent review article (by G. M. Clemence) in the Explanatory Supplement to the Ephemeris (p. I68-174) and of the classical paper by W. de Sitter (edited and completed by Dirk Brouwer) in Bull. Astron. Inst. Netherlands, vol. 8, 1938, p. 2 13-23 г.

1. I.2. I take the viewpoint that the purpose of adopting a system of astronomical constants is to ensure that the fundamental astronomical ephemerides of high precision are computed on a uniform and rigorously defined basis over long periods of time. I deliberately ignore the important, but quite distinct, problem of the choice of constants that will give the " best" ephemerides over short periods of time, such as are required for space research. I ignore also constants of astrophysical interest.

1. 1.3. In order to demonstrate the practicability of the new system I have given a typical numerical value for each constant, but neither these values nor the rather pedantic notation that I have used are essential parts of the system. I have not made any attempt to choose the optimum values for the fundamental constants; in general I have merely used values that are reasonably close to the current observed values.

1.I.4. I have also expressed my present views on the desirability of amending the current system and on the ways in which any changes could be put into effect. I consider, however, that these views are the least significant part of this review.

1. I.5. In preparing this material for publication in the proceedings of the Symposium I have made a few minor changes in the text and in the numerical values. I have omitted entirely the original lengthy sections on precession and nutation and on time since these topics were not discussed in detail at the symposium.

1.2. Principles of construction of the system. - 1.2. I. In constructing a system of astronomical constants, we have two separate tasks. First of all, we have to define the relationships between the constants, and then we have to adopt particular values for each constant. The adopted numerical values should be such that their agreement with the determinations of all directly observable constants is as good as 
the restraints (imposed by the relationships between the constants) permit. It is advantageous if the differences between the adopted and observed values for each constant are so small that the ephemerides can be adjusted by the application of only first-order corrections; it seems likely that this can be readily achieved once agreement on what is the observed value has been reached.

1.2.2. When we come to define the relationships between the constants we realize that there are three kinds of constants, to which we can refer by the names absolute, fundamental and derived constants. The value of an absolute constant is fixed once-and-for-all, and essentially serves to define one quantity; Gauss' constant of gravitation is one such constant and the number of ephemeris seconds in the tropical year at rgoo is another. The adopted values of the fundamental constants may be chosen independently of each other; but the adopted values of the derived constants are then determined by the consistency relations between the constants.

1.2.3. A particular system is defined by the assignment of the various constants to each of the three types and by the statement of the consistency relations. There is no unique way of separating the fundamental and derived constants - or even of deciding which constants shall be considered to be part of the system. The fundamental constants need not necessarily be quantities that are directly and accurately observed. It is perhaps more important that they should be simply and rigorously defined, and that the resulting expressions for the derived constants shall be as straightforward as possible. It is also an advantage if the constants of the system can be defined without reference to any particular external theory, i. e. quantities peculiar to one method of development should be avoided. In the absence of good reasons to the contrary the perpetuation of constants in current use is to be preferred to the introduction of new ones.

1.2.4. The notation is more complicated than that in current use since for the purposes of this review it is necessary to avoid ambiguity and to specify the units to which the numerical values refer. The units are usually indicated by superscripts as follows : $\mathrm{m}$, metre; , astronomical unit (a. u.); r, georadius (see 2. 1.3); ", seconds of arc; and s, (ephemeris) seconds. The following subscripts are used to indicate the object or type to which the main symbol applies : e, Earth; $x$, Moon; s, Sun; ${ }_{b}$, barycentre of the Earth and Moon; and ${ }_{L}$, light. In specifying the (typical) numerical value of each constant the following notation is used : $\equiv$ før absolute constants; = for fundamental constants; $\rightarrow$ for derived constants. The numerical values of the absolute and fundamental constants are treated as exact, and fictitious zeros are added when 
necessary before the values of derived constants are calculated. The numerical values of mathematical constants occurring in the relationships are taken to sufficient figures to give numerical consistency.

2. Survey of the system. - 2. I. SCale and SPEed in the solar SYSTEM. - 2. I. I. The principal purpose of the constants of this section is to define the relationships between the various units of length that are used for measures of distance in the solar system. However, since interplanetary distances can now be measured indirectly by radar techniques it is necessary to include the velocity of light; this in turn introduces the constant of aberration.

2. I.2. The fundamental unit of length that is used in the theories of the heliocentric motions of the planets is the astronomical unit (a. u.). It is defined by taking the Gaussian gravitational constant $(k \equiv 0.017202098950)$ to be a certain exact number (i. e. as an absolute constant) when the unit of mass is the mass of the Sun, the unit of length is the astronomical unit, and the unit of time is the ephemeris day. In these units Kepler's third law may be expressed as

$$
n^{2} a^{3}=l^{2}(\mathrm{I}+\mathrm{l}),
$$

where $n$ is the mean angular sidereal motion in radians per day, $a$ is the unperturbed (or Keplerian) mean distance, and $M$ is the mass of the planet. The sidereal period of a particle of negligible mass moving around the Sun in an unperturbed circular orbit of radius $i$ a. u. would be $\frac{2 \pi}{l_{i}}$ ephemeris days or I Gaussian year; such an orbit we shall call a Gaussian orbit. The mean distance of the Earth from the Sun is just greater than i a. u.

2. I.3. In classical astronomy, distances are measured indirectly through parallactic angular shifts due to the displacement of the observer from the centre of the Earth. The solar parallax $\left(\pi_{\odot}\right)$ is defined to be the equatorial horizontal parallax of an object at a distance of $r$ a. $u$. It therefore defines the ratio of the astronomical unit to the equatorial radius of the reference spheroid for the Earth, which we shall call the georadius; this ratio is here denoted by $L_{.1}^{r}$ and is given by

$$
L_{\mathrm{il}}^{\mathrm{r}}=\operatorname{cosec} \pi_{\odot}=\frac{206264.8}{\pi_{\odot}^{\prime \prime}}
$$

If the number of metres in the georadius is taken as a fundamental constant and is denoted by $r_{0}^{\mathrm{m}}$, then the number $\left(\mathrm{L}_{\mathrm{a}}^{\mathrm{m}}\right)$ of metres in $\mathrm{r}$ a. $\mathrm{u}$. is given by

$$
\mathrm{L}_{\mathrm{a}}^{\mathrm{m}}=r_{10}^{\mathrm{m}} \mathrm{L}_{\mathrm{i}}^{\mathrm{r}}
$$


2. 1.4. In radar astronomy distances are measured indirectly by the time of travel of electromagnetic waves in the interplanetary medium. Since the velocity of light (i. e. speed of propagation of electromagnetic waves in vacuo) can be measured accurately in metres per second it is convenient to take this as one of the fundamental constants. The velocity of light in metres per second is denoted here by $\mathrm{V}_{\mathrm{L}}^{\mathrm{m}}$; the difference between the atomic and ephemeris seconds can be ignored. The observation of the travel time to a planet whose distance is known in astronomical units therefore leads to a determination of $L_{i}^{m}$, and hence to $\pi \odot$ if the adopted value of $r_{0}^{\mathrm{m}}$ is used. Even though the solar parallax is now accurately determined (via $\mathrm{V}_{\mathrm{L}}^{\mathrm{m}}$ and $r_{0}^{\mathrm{m}}$ ) from such radar observations (and those based on Doppler shifts), it is here retained as a fundamental constant, but $\mathrm{L}_{.1}^{\mathrm{m}}$ would be a suitable alternative. There does not seem to be any advantage in introducing the light-second as an additional unit of distance; corrections for any difference between the actual speed of propagation and the adopted value can easily be applied to observed or predicted values of the travel time.

2. I.5. The constant of aberration ( $\%$ ) is usually defined as the ratio of the mean velocity of the Earth to the velocity of light but it is simpler to define it as the ratio $\left(\varkappa_{0}\right)$ of the speed of a particle in a Gaussian orbit (of $\mathrm{I}$ a. $u$. radius) to the velocity of light. The difference between these two definitions is much smaller than the errors in the observational determination of the aberrational displacements in the positions of stars, and can easily be taken into account in the reduction of observations. The use of this new definition simplifies the statement of the relationships in the system and avoids the need to introduce such quantities as the eccentricity of the Earth's orbit. The constant of aberration $\left(x_{0}\right)$ defined in this way is derived from the other constants by the relations

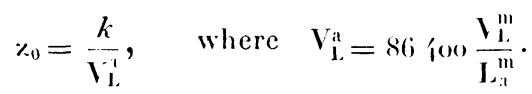

The intermediate quantity $V_{\mathrm{L}}^{i}$ is the velocity of light expressed in a. $u$. per day. The constant of aberration is usually expressed in seconds of arc and is then given by

$$
\varkappa_{0}^{\prime \prime}=206264.8 x_{0}=\frac{3548 \cdot 18-66}{V_{L}^{\prime}} .
$$

An auxiliary quantity used in calculating planetary aberration is the light-time for unit distance; its value in seconds of time is given by

$$
\mathrm{T}_{\mathrm{i}}^{\mathrm{s}}=\frac{86400}{\mathrm{~V}_{\mathrm{L}}^{\mathrm{i}}}=\frac{\mathrm{l}_{\mathrm{i}}^{\mathrm{m}}}{\mathrm{V}_{\mathrm{L}}^{\mathrm{m}}}
$$


2. 1.6. A simple check on the numerical consistency of the constant of aberration and the adopted values of the velocity of light, the solar parallax, and the metric measure of the georadius is afforded by noting that

$$
\varkappa_{0}^{\prime \prime} \mathrm{V}_{\mathrm{L}}^{\mathrm{m}} \frac{\pi_{\odot}^{\prime \prime}}{r_{0}^{\prime \prime \prime \prime}}=8470.673 \ldots
$$

This is an absolute constant depending only on the value of the absolute constant $k$ and some mathematical constants.

2.1.7. For illustrative purposes we may adopt the following values for the fundamental constants :

for the solar parallax, in seconds of arc,

$$
\bar{\tau}_{\odot}^{\prime \prime}=8.79415
$$

for the georadius, in metres,

$$
r_{0}^{m}=6378 \mathbf{I} 65
$$

and for the velocity of light, in metres per second,

$$
\mathrm{V}_{\mathrm{L}}^{\mathrm{m}}=29979^{2} 500 .
$$

We then obtain the following values for the derived constants :

for the astronomical unit, in georadii,

$$
L_{\mathrm{it}}^{r} \rightarrow 23454.77
$$

for the astronomical unit, in metres,

$$
\mathrm{I}_{\mathrm{a}}^{\mathrm{m}} \rightarrow \mathrm{I} / 49598.4 \times \mathrm{IO}^{\mathrm{li}} ;
$$

for the velocity of light, in a. u. per day,

$$
V_{L}^{a} \rightarrow 173.144
$$

for the constant of aberration, in seconds of arc,

$$
x_{0}^{\prime \prime} \rightarrow 20.4927
$$

and for the light-time for unit distance, in seconds per a. u.,

$$
\mathrm{T}_{\mathrm{a}}^{\mathrm{s}} \rightarrow 499 \cdot 007 \text {. }
$$

The roles of $\tau_{\odot}^{\prime \prime}$ and $\mathrm{L}_{\mathrm{a}}^{\mathrm{m}}$ may be interchanged.

2.2. Figure and Gravity-field of the Earth. - 2.2. I. Astronomers are interested in the figure, structure and gravitational field of the Earth for the following distinct reasons. 
(a) A knowledge of the figure is necessary for the parallactic reduction between topocentric and geocentric positions.

(b) A knowledge of the structure (i. e. distribution of density, especially the relative sizes of the moments of inertia, the elasticity, etc.) is necessary for the theoretical study of the luni-solar precession and nutation.

(c) A knowledge of the gravitational field is required in the theories of the motions of bodies (including the Moon) in the neighbourhood of the Earth.

Ideally, the figure and the field should be deduced rigorously from a model of the structure of the Earth and so would form a completely self-consistent system. Similarly, the luni-solar precession and the nutation should be deduced from this model and the theories of motion of the Sun and Moon. However, the incomplete state of our knowledge of the structure of the Earth and the comparatively crude state of the development of the theories of precession and nutation imply that we are not likely to attain this ideal situation in the near future.

2.2.2. It can even be argued strongly that the figure and the gravity field should be treated quite independently, i. e. that astronomers should adopt the " best" values of the coefficients in the expansion for the external gravitational field and of the geocentric co-ordinates of astronomical observatories. However, this would give only a marginal increase in accuracy at the expense of inconsistency with the constants used in geodesy. In fact it seems that astronomers should endorse the recent recommendations of the International Gravity Commission covering the relationships between the adopted values of the flattening of the reference spheroid (whose meridional section is an ellipse), the coefficients in the formula for apparent gravity on this spheroid, and those in the expansion for the external field. These recommendations depend on the well-established result that it is possible to choose the coefficients in the expansion for the external field in such a way that the reference spheroid is an equipotential surface (i. e. so that apparent gravity is normal to the reference spheroid). Further it now appears that for an appropriate value of the flattening these coefficients are in reasonable agreement with the values deduced from observations of the motions of artificial satellites.

2.2.3. The relationships are simplest if we take as fundamental constants $: r_{0}^{\mathrm{m}}$, the georadius, $i$. e. the equatorial radius of the reference spheroid, in metres; $g_{0}^{\mathrm{m}}$, the apparent acceleration, in metres per second, on the equator of the reference spheroid; $f$, the flattening of the reference spheroid; and $m_{A}$, the ratio of the mass of the Earth's atmosphere to the mass of the Earth (including atmosphere). In addition (), the rate of rotation of the Earth in radians per second, enters into the formulae, but its value is not required accurately for this purpose. 
2.2.4. The expression for the external gravitational potential of the Earth (including the atmosphere) may be written in the form

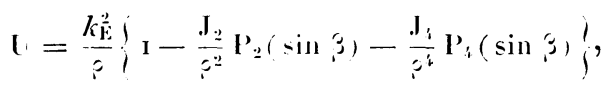

where $k_{\mathrm{t}}$ is the Gaussian-type gravitational constant for the Earth when the unit of mass is the mass of the Earth, the unit of length is the georadius, and the unit of time is the ephemeris second;? is the geocentric distance; and $\beta$ is the geocentric latitude. (In the Explanatory Supplement to the Ephemeris the geocentric latitude is denoted by $\left.\varphi^{\prime}\right)$. $\mathrm{P}_{2}$ and $\mathrm{P}$; are the Legendre polynomials for which

$$
P_{2}\left(x^{2}\right)=\frac{1}{3}\left(3 x^{2}-1\right) \quad \text { and } \quad P_{1}\left(x^{2}\right)=\frac{1}{8}\left(3 i x^{2}-30 x^{2}+3\right)
$$

A term in $\mathrm{J}_{i}$ is necessary if the expression for $\mathrm{L}$ is to be correct to terms of order $f:$. The odd-order coefficients $\mathbf{J}_{: ;}, \mathbf{J}_{;}, \ldots$ and the coefficients of the terms dependent on longitude are zero for a spheroid. For the purpose of astronomy there does not seem to be any advantage in introducing a triaxial ellipsoid instead of a spheroid, although King-Hele

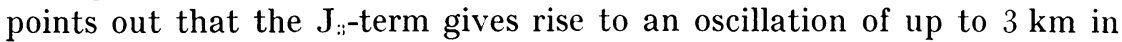
the perigee distance of the Moon.

2.2.5. The expression for the apparent acceleration on the Earth's surface at geodetic latitude $?$ may be written in the form

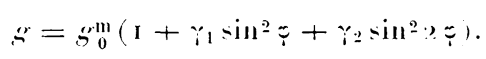

A term in $\sin ^{2} 0 \sin ^{2} 20$ is necessary if the development is to be correct to terms of order $f^{\prime:}$.

2.2.6. Expressions for $\mathrm{J}_{2,}, \mathrm{~J}_{i}$ and $\dddot{\imath}_{1}, \gamma_{2}$ in terms of the flattening $f$ and an auxiliary parameter $\%$ have been deduced recently by A. H. Cook (Geophys. J., vol. 2, I gj9, p. I99-2 I 4) and are here quoted only to terms of order $f$. The auxiliary parameter $q$ is dependent on the rate of rotation and is defined by

$$
q=\frac{(1)^{2}}{k_{\mathrm{E}}^{2}}(\mathrm{I}-f)\left(1+m_{1}\right):
$$

it has to be calculated by iteration, since $k_{\mathrm{F}}^{*}$ is derived from the expression

$$
k_{i}^{2}=\left\{\frac{g_{0}^{m}+r_{0}^{m}(1)^{2}}{r_{0}^{m}}\right\}\left\{\frac{1+m_{1}}{I+\frac{3}{2} J_{2}-\frac{1 j}{g} J_{i}}\right\} .
$$

which itself involves $J_{2}$ and $J_{1}$. This expression for $k_{:}^{*}$ is deduced by considering the field at the equator where both the geocentric and geodetic latitudes are zero. The correction for the mass of the atmosphere is 
small, but must formally be taken into account. The coefficients in the expressions for $\mathrm{L}$ and $g$ are given by

$$
\begin{aligned}
& J=\frac{m}{3} f-\frac{1}{3} q-\frac{1}{3} f=\frac{2}{3} f q . \\
& \mathrm{J}=-\frac{1}{j} f=+\frac{i}{3} \cdot f q \text {. } \\
& \dddot{r}_{1}=\frac{j}{i} q-j+\frac{1 j}{i} q^{2}-\frac{1 j}{1 i} f q . \\
& \gamma_{2}=\frac{\mathbf{I}}{8} \cdot f-\frac{5}{8} f q .
\end{aligned}
$$

In practice the value of $f$ will be chosen so that the derived value of $J_{2}$ is in good agreement with observation.

2.2.7. For comparison with other systems it is useful to include $r_{::}^{\prime \prime \prime}$, the polar radius of the Earth in metres, and $r_{1}^{\prime \prime \prime}$, the mean radius of the Earth in metres; these are given by

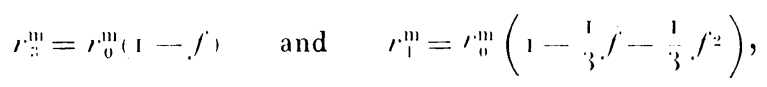

where $r_{1}^{\mathrm{m} \prime \mathrm{l}}$ refers to the geocentric latitude $\operatorname{arc} \sin \frac{1}{\sqrt{3}} \cdot$ It has been suggested that the mean radius rather than the equatorial radius should be treated as a fundamental constant since it is better determined and leads to simpler formulae; however, this change from current practice is hardly justified since the latter claim is itself disputed and since there are at least three possible definitions of the mean radius.

2. ..8. The introduction of the quantity $k_{\mathrm{k}}$ serves a useful purpose in problems on geocentric orbits, and this quantity can also be considered to define a unit of length similar to the astronomical unit. This unit of length is the radius of an equatorial circular orbit in which a particle of negligible mass and free of perturbations would revolve round the Earth in a period of $\frac{\pi}{k_{\mathrm{t}}}$ ephemeris seconds. This unit of length can, however, only differ from the georadius by a very small amount. Confusion is likely to be caused if such an extra unit of length is explicitly introduced into the system or if, as has been suggested, the value of $k_{\mathrm{r}}$ is treated as an absolute constant that is not necessarily consistent with the values of the related constants.

An additional quantity corresponding to $k_{\mathrm{F}}^{2}$ is the gravitational constant for the Earth when the unit of length is the metre and not the georadius; it is given by

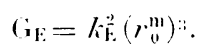


2.2.9. For illustrative purposes we may adopt the following values of the fundamental constants :

for the georadius (equatorial), in metres,

$$
r_{0}^{\mathrm{m}}=6378 \times 65
$$

for the apparent gravity (equatorial), in metres per second squared,

$$
g_{0}^{\mathrm{m}}=9.78049
$$

for the flattening of the reference spheroid,

$$
f=\frac{1}{298.25}
$$

for the relative mass of the atmosphere,

$$
m_{\mathrm{A}}=0.000 \mathrm{00I} \text {; }
$$

and for the rate of rotation of the Earth, in radians per second,

$$
\text { (1) >0.000 07? 9?1. }
$$

We then obtain the following values for the derived constants :

for the geocentric gravitational constant, in georadii and seconds,

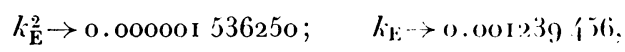

for the constants in the expression for surface gravity,

$$
\gamma_{1} \rightarrow+0.00 i 3020, \quad \gamma_{2} \rightarrow-0.00000 ; 8 \text {; }
$$

for the coefficients in the expression for the external gravitational potential,

$$
\mathrm{J}_{2} \rightarrow+0.0010827, \quad \mathrm{~J}_{1} \rightarrow>-0.0000024 ;
$$

for the mean and polar radii of the Earth, in metres,

$$
r_{1} \rightarrow 637 \mathrm{r} \text { or } 3, \quad r_{3} \rightarrow 6356>80
$$

and for the geocentric gravitational constant, in metres and seconds,

$$
\mathrm{G}_{\mathrm{E}} \rightarrow 398.6 \mathrm{IOg} \times 10^{12} .
$$

2.3. Constants of the Earth-Moon system. - 2.3. I. This subsystem is concerned with the principal constants defining the motion of the barycentre of the Earth and Moon around the Sun and the motion of the Moon around the Earth. The principal difficulties arise from the possible ambiguity in the use of the term " mean distance" and from the multiplicity of parallax constants. These difficulties are largely overcome by the use of the mean sidereal motion as the fundamental constant 
REVIE IV OF TIIE SYSTEII OF ASTRONOMICAL CONSTANTS. 261

while making mean distance a derived constant calculated from the appropriate form of Kepler's third law. Other constants required in any particular theory can be derived from this Keplerian (or unperturbed) mean distance by relations that should be explicitly stated in the theory. For example, the Keplerian mean distance $a_{\mathrm{B}}^{\mathrm{a}}$ of the Earth-Moon barycentre from the Sun, in astronomical units, is determined from

$$
\left(a_{\mathrm{B}}^{\mathrm{a}}\right)^{:}=\frac{\mathrm{l}^{2} \cdot\left(\mathbf{I}+\mathbf{M}_{\mathbf{B}}\right)}{n_{\mathrm{B}}^{-\frac{1}{\mathrm{~B}}}},
$$

where $\mathrm{M}_{\mathrm{k}}$ is the combined mass of the Earth and Moon, in units of the Sun's mass, $n_{\mathrm{B}}=\frac{n_{\mathrm{B}}^{\prime \prime}}{206264.806}$ and $n_{\mathrm{B}}^{\prime \prime}$ is the sidereal mean motion of the Sun (or barycentre) in seconds of arc per (ephemeris) day; but $a_{13}^{i}$ is not the same as the constant term in the series for the radius vector since an additional constant term arises from the planetary perturbations.

2.3.?. The mass of the Earth in units of the mass of the Sun is determined by the consistency relations that exist between it, the solar parallax $\left(\tau_{0}\right)$, the Gaussian gravitational constant $(k)$ and the corresponding constant $k_{1:}$ (or $\mathrm{G}_{\mathrm{l}}$ ) for motion about the Earth. Thus we have

$$
k_{\mathrm{E}}^{2}=\frac{k^{2}(\text { No. of georadii in I a.u. })^{3}}{(\text { Mass of Sun in units of Mass of Earth) (No. of seconds in I day.2 })^{2}}
$$

and, if $\mathrm{M}_{\mathrm{E}}$ is the mass of the Earth in units of the Sun's mass,

$$
\mathrm{I}_{\mathrm{H}}=(86400)^{2}(\sin \pi \odot)^{3} \frac{k_{\mathrm{E}}^{2}}{k^{2}} .
$$

It is convenient to take $m_{3}$, the mass of the Moon in units of the Earth's mass, as another fundamental constant. It then follows that the combined mass of the Earth and Moon, in units of the Sun's mass, is given by

$$
\mathrm{M}_{\mathrm{B}}=\mathrm{M}_{\mathrm{E}}\left(\mathrm{I}+m_{\mathrm{M}}\right)
$$

2.3.3. The Keplerian mean distance $a_{\mathrm{M}}$ of the Moon from the Earth in astronomical units is given by

$$
\left(a_{\mathbf{M}}^{a}\right)^{3}=\frac{k^{2} \mathbf{M}_{\mathbf{B}}}{n_{\mathbf{M}}^{2}}
$$

where $n_{M}=\frac{n_{M}^{\prime \prime}}{206264.806}$ and $n_{M}^{\prime \prime}$ is the sidereal mean motion of the Moon in seconds of arc per (ephemeris) day. Similarly, the Keplerian mean distance of the Moon from the Earth in georadii is given by

$$
\left(a_{\mathrm{M}}^{\mathrm{r}}\right)^{3}=\frac{k_{\mathrm{E}}^{2}\left(\mathrm{I}+m_{\mathrm{MI}}\right)(86400)^{2}}{n_{\mathrm{I}}^{2}} .
$$


These two measures of the mean distance are connected by the relation

$$
a_{\mathrm{M}}^{\mathrm{r}}=a_{\mathrm{M}}^{i \mathrm{~L}} \mathrm{~L}_{\mathrm{il}}^{\mathrm{r}}=a_{\mathrm{m}}^{i \mathrm{t}} \operatorname{cosec} \bar{\sigma}_{\odot} \text { : }
$$

where $L_{i i}^{r}$ is the number of georadii in 1 a. u. and $\pi$ : is the solar parallax.

The Keplerian mean distance in metres is given by

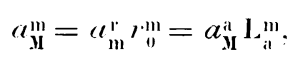

where $r_{0}^{\mathrm{m}}$ and $\mathrm{L}_{\mathrm{a}}^{\mathrm{m}}$ are the number of metres in I georadius and I a. u., respectively.

The (Keplerian) mean lunar parallax $\tau_{M}$ corresponding to $a_{N}$ is given by

$$
\sin \pi \mathrm{M}=\frac{r_{u}^{m}}{a_{\mathrm{M}}^{\mathrm{m}}}=\frac{1}{a_{\mathrm{M}}^{\mathrm{r}}}=\frac{\sin \pi}{u_{\mathrm{M}}^{i}}
$$

and its value in seconds of arc by

$$
\pi_{M}^{\prime \prime}=200 \cdot 2, \frac{1}{3} 3 \sin \pi_{N} \text {. }
$$

2.3.4. Brown's theory of the motion of the Moon is not developed in terms of the perturbations of an elliptic orbit, but instead Brown uses Hill's variational orbit (which approximates to the motion of the Moon as perturbed by the Sun). He obtains an expression for the sine parallax and finally adopts a value for the constant term in this expression; he states that his theory gives 1.0009076 for " the ratio of the constant term in the final expression for the inverse of the Moon's radius vector to ", where $n^{2} a^{3}=\mathrm{E}+\mathrm{M}$ " (M.N.R.A.S., vol. 75, г 915 , p. $\left.\check{1} 16\right)$. Hence, denoting the constant term in sine parallax by $\sigma_{\mathrm{I}}^{\prime \prime}$, it is reasonable provisionally to adopt the relationship

$$
\sigma_{M}^{\prime \prime}=1.00090-6 \times 206264.8 \sin \pi_{M}=1.000861 ; j-\pi_{M}^{\prime \prime}
$$

The corresponding mean distance of the Moon in metres is given by

$$
a_{11}^{\mathrm{m}}=\frac{a_{\mathrm{M}}^{\mathrm{m}}}{1.0009076}
$$

A much more detailed study of Brown's theory is required if the fundamental lunar ephemeris is to be brought into conformity with the system of astronomical constants.

2.3.5. It was suggested above that $m_{\mathrm{v}}$, the mass of the Moon in units of the Earth's mass, should be taken as a fundamental constant and it was shown how this mass ratio enters into the relationships between, for example, the lunar and solar parallaxes. It also enters into two other quantities that may be compared with observations; these are 
the lunar inequality in the Sun's longitude and the parallactic inequality in the Moon's ecliptic longitude; they may be defined as the coefficients of $\pm \sin D$ (where D is the elongation of the Sun from the Moon) in the expressions for the longitudes. The lunar inequality in the Sun's longitude is a geometrical effect due to the monthly motion of the Earth round the barycentre, and the same type of monthly parallactic displacement is accurately observable in the motions of minor planets; it is therefore convenient to define the constant of lunar inequality $I_{M}$ by the relation

$$
\mathrm{I}_{M}=\frac{m_{M}}{\mathrm{I}+m_{M}} \frac{\sin \pi_{\odot}}{\sin \pi_{M}} .
$$

This relation may be written as

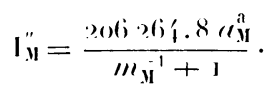

In the absence of any similar standardisation for the constant of parallactic inequality $I_{:}$, it is necessary to use the relation applicable to Brown's theory, namely

$$
\begin{aligned}
I_{\mathrm{P}}^{\prime \prime} & =498 ; 3, \frac{1-m_{\mathrm{M}}}{1+m_{\mathrm{M}}} \frac{\sin \pi_{\odot}}{\sin \pi_{\mathrm{M}}} \\
& =0.24169 j\left(m_{\mathrm{M}}^{-1}-1\right) I_{\mathrm{M}}^{\prime \prime} .
\end{aligned}
$$

2.3.6. For illustrative purposes we may adopt the following values of the fundamental constants in addition to those given above :

for the mass of the Moon, in units of the Earth's mass,

$$
m_{M}=\frac{1}{x_{1} \cdot 31}
$$

and for the sidereal mean motion of the Moon, in seconds of arc per day,

$$
\left.n_{.1}^{\prime \prime}=\text { 仁 } 13\right\}^{\prime \prime} .88_{9} 8 ; 1 .
$$

We then obtain the following values for the derived constants :

for the mass of the Earth, in units of the Sun's mass,

$$
\mathbf{M}_{\mathbf{E}} \rightarrow \frac{1}{3329 \underline{1} \mathbf{1}}
$$

for the mass of the Earth + Moon, in units of the Sun's mass,

$$
I_{\mathrm{B}} \rightarrow \frac{\mathrm{I}}{3: 28896}
$$


for the Keplerian mean distance of the Moon from the Earth, in astronomical units, georadii and metres, respectively,

$$
a_{\mathbf{M}}^{\mathrm{n}} \rightarrow 0.0025 ; \mathrm{I} 89 . \quad a_{\mathrm{M}}^{\mathrm{r}} \rightarrow 60.3232, \quad a_{\mathrm{M}}^{\mathrm{m}} \rightarrow 38.1 .55 \mathrm{I} \times \mathbf{1} 0^{\mathrm{i}}:
$$

for the Keplerian mean lunar parallax, in seconds of arc,

$$
\pi_{M}^{\prime \prime} \rightarrow 3 \text { irg.49; }
$$

and for the constant of lunar inequality, in seconds of arc,

$$
I_{M} \rightarrow 6^{\prime \prime}, 4450 \text {. }
$$

We also obtain the following derived constants on the basis of Brown's theory of the motion of the Moon :

for the constant term in sine parallax, in seconds of arc,

$$
\sigma_{\mathbf{M}}^{\prime \prime} \rightarrow 3 \text { 价".4亿; }
$$

for the mean distance of the Moon, in metres,

$$
a_{\mathrm{II}}^{\mathrm{m}} \rightarrow 38 \mathrm{i} \cdot \mathrm{1} 02 \times \mathrm{IO}^{\mathrm{k}} ;
$$

and for the constant of parallactic inequality, in seconds of arc,

$$
I_{P}^{\prime \prime} \rightarrow I 0 j^{\prime \prime} .101 \text {. }
$$

2.4. Other constants. - 2.1.1. The fundamental constants of precession and nutation may be chosen in one of two distinct ways. Either the constants may be chosen so that they define immediately the transformation from a true co-ordinate system of any date to a fixed co-ordinate system corresponding to some standard epoch, or they may be chosen (as at present) so that they define the separate constituents that contribute to the total motions of the co-ordinate systems. Ideally the choice should be immaterial since it should be possible to determine either set of constants from the other, but at present it seems that a precise, direct statement of the relationships concerned does not exist, and it is not even certain that the two sets of constants in current use are in fact precisely equivalent. (We hope to discuss these and related matters in a separate paper.) The dependence of the constants of precession and nutation on other quantities such as the mechanical ellipticity of the Earth is not sufficiently strong or certain to justify the inclusion of additional relations in the system.

2.4.2. The fundamental expression on which depend the definition of ephemeris time and all the other constants concerned with time is that for the geometric mean longitude of the Sun. The derivation from it of the expression for the right ascension of the fictitious mean Sun, which is used in calculating universal time from the observed sidereal 
time, involves the constant of aberration and the general precession in right ascension, as well as the change of the independent variable from ephemeris time to universal time.

2.4.3. The only other constants that may be deemed to be members of a system of fundamental constants are the masses of the planets in units of the mass of the Sun. Ideally, the same masses should be used in the theories of motion of all planets (and satellites) and in the theory of precession etc., but in practice small differences from theory to theory may not be significant. It would be useful to draw up a table of the values that have actually been used and to adopt a set that best matches the values used in recently developed theories.

2.4.4. There are many other quantities that can be considered to be astronomical constants but there appear to be few, if any, consistency relations to be satisfied between them, and the fields in which they are used are practically independent of each other. However, if only to avoid an increasing number of different values, it would be useful to list agreed values of the relative masses of the satellites and the J-coefficients, apparent flattenings and semi-diameters at unit distance of the planets. In addition the constants concerned with the gravity field, figure and libration of the Moon should be specified; the relation of Watt's reference surface for the limb-correction charts to the nominal semi-diameter of the Moon should also be precisely defined in the system.

3. Comments on the introduction of a new system. - 3. . The desirability and procedure for change. - 3. I. I. The system in current use has been developed from the agreements reached at the meetings of the directors of the national ephemerides in 1896 and $191 \mathrm{r}$, and is to a large extent based on Newcomb's discussion. The system has several disadvantages : it is not clearly defined; the adopted values are not internally consistent; and some of the adopted values are not in good agreement with recent determinations. The main virtue of the current system is that much of it has been in continuous use for over sixty years in the fundamental ephemerides. Moreover, ephemerides based on the present system are in print or proof up to the year 1968 ; they have been computed in their entirety up to the year I97 I and most of the computing has been done for the years r972-1980. Newcomb's system of precessional motions is also embodied in several important star catalogues, such as the Albany General Catalogue, the FK3 and FK4, the Zodiacal Catalogue, etc. For many of these ephemerides and catalogues the effect of changes in the adopted constants would be quite small in relation to the existing effects of deficiencies of the underlying theories or positions. Hence there would often be no significant 
increase in the accuracy of the ephemerides or star positions and motions if they were to be recomputed using better constants.

3. I. . However, I consider that the time is now ripe for agreement on the eventual introduction of a new system that does not suffer from the above disadvantages, and I have indicated in section 2 the form that I consider would be appropriate. On matters of detail I realize that the system requires further scrutiny, and possible amendment, but I do feel that it can be developed into a clearly defined and internally consistent system in which the values of the constants, both fundamental and derived, are in reasonable agreement with observation. There are several reasons why I consider that the decision to change to a new system should not be deferred further and that the new system should be prepared in time for consideration and adoption at the twelfth General Assembly of the I. A. U. in 1964. These reasons include : (a) The introduction of electronic computers has led to the speedier completion of new theories of motion of the planets and of the ephemerides derived from the theories, and to the possibility of a re-reduction of past observations on a uniform basis. It is desirable that this work should be done using constants that are self-consistent and as close to the truth as possible so that second-order errors are quite negligible. (b) The introduction of new techniques for measurement and study of the solar system has already drawn attention to the deficiencies of the present system and to the need, for practical purposes, of better ephemerides. (c) Consideration is now being given to the possibility of printing The Astronomical Ephemeris by new techniques and of introducing changes of both content and arrangement at the same time. The extent of such changes would obviously be conditioned by the prospects for changes in the basis of the ephemerides.

3. г.3. Since it is probable that even in a new system there would be no significant change in the precessional constants, it is sufficient to consider only the manner in which a new system could be introduced into the fundamental ephemerides. There could be three distinct stages, as follows. (a) Precepts for the correction of ephemerides already in print to be published. (b) Ephemerides based on the new system but the present theories to be computed for current and future years (say, from 1960 to 1980 ) and made available (on a restricted basis) in machine-readable form (e. g. magnetic tape) or even as machine-listings. They could be introduced into the almanacs as soon as is economically feasible, but this is unlikely to be until The Astronomical Ephemeris for 1972 (copy for which must be sent to the printer in 1966). (c) Ephemerides based on the new system and new theories (or elements) to be computed whenever possible; these could be introduced into the almanacs but could also be published separately for many years at a time. Since 
the first part of the Ephemeris has to be printed so long in advance, it is possible that some items in $(c)$ might be available before the first almanac based on the new system.

3. 2. Comparison OF THE CURRENT AND PROPOSED SyStems. - 3.2. I. It must first of all be emphasised that the particular values given in the text in section 2 are only intended to illustrate the system of relationships and that no attempt has been made to choose the best values for the fundamental constants.

3.2.2. In the proposed system of constants concerned with scale and speed in the solar system the constant of aberration is treated as a derived quantity. The solar parallax is still retained as a fundamental constant, although its place could be taken by the measure of $\mathrm{I}$ a. u. in metres or by the velocity of light in a. u. per day. (The latter is the quantity actually obtained from radar travel-time experiments.) The need to obtain internal consistency and the desirability of introducing better values for, say, the solar parallax implies that practically all of these constants will be changed. Any likely values of the fundamental constants should give an adequate value for the constant of aberration, whose observational determination appears to be fraught with systematic errors.

3.2.3. For the constants defining the figure and gravity field of the Earth, we have essentially followed the recommendations of the International Gravity Commission, i. e. the external gravity field is to be such that the reference spheroid is everywhere normal to the apparent gravity. A change in the adopted value of the flattening (now 1/297) will be required if, as is recommended, its value is chosen so that the corresponding value of $\mathrm{J}_{2}$ agrees with the value deduced from observations of artificial satellites. (The value of $J_{2}$ would be in error by almost ${ }_{1} \%$ if the present figure were to be retained.) The formulae are, here, given only for the second-order analysis; the additional precision of the thirdorder formulae hardly justifies their extra complexity. For most astronomical purposes the derived value of $J_{4}$ (with zero values for the other coefficients) appears to be of adequate accuracy. The practical consequences of a change in the figure are not likely to be extensive in astronomy and first-order corrections should suffice. The I. A. U. should perhaps make known its views on these constants to the I. U.G.G., but should then follow any recommendations made by I. U. G. G.

3.2.4. The constants for the Earth-Moon system are inadequately treated in the current system even though they are of fundamental importance. The principal faults in the current system are that two different values for the masses of the Earth and Moon are used and the term lunar parallax is not used in any clearly defined sense. The only 
new feature of the proposed system is the explicit introduction of the Gaussian-type gravitational constant $k_{\mathrm{E}}$ for motion around the Earth; it is, however, obtained as a derived constant and it is not intended that it should be treated as an absolute constant as is the Gaussian constant $k$. There seems to be no good reason to perpetuate the inconsistencies of the present system, nor should there be any difficulty in finding a consistent set of adequate accuracy.

3.2.5. Unless greater mathematical precision is required we can ignore the minor inconsistencies among the various precessional coefficients in current use. Further there is little to recommend any attempt to improve the basic coefficients since any gain in accuracy would not justify the heavy price that would be required to adjust systematically the many star catalogues based on the current system.

3.2.6. Even at the present time the expressions used for the geometric mean longitude of the (true) Sun (in ephemeris time) and the right ascension of the fictitious mean Sun (in universal time) are not strictly consistent. However, since universal time is not a fundamental system of time, the minor illogicalities in its definition can be allowed to persist; they are unlikely to lead to any significant discrepancies before the next review of the system! Perhaps by then a more direct system of defining time for use in civil life and navigation will have been devised.

3.2.7. Of the many other constants required to define completely the dynamical and geometric properties of the solar system there is little that need be said here. Clearly the Symposium cannot hope to consider all these in detail, but one person, or small group of persons, could be given the task of sifting the evidence and presenting a list of values to the I. A. U. The national ephemerides could be brought into line with the adopted list at the earliest opportunity thereafter.

3.2.8. The principal differences between the current and the proposed systems lie in the explicit statement of the consistency relations between the constants and in the selection of the fundamental constants from the totality of constants concerned. It is considered, however, that the introduction of such a consistent system will be of sufficient benefit to astronomy to justify the effort required to make the system a practical reality.

Acknowledgment. - I wish to acknowledge the assistance given by Miss D. E. Hutchings in the preparation of this review and the helpful criticisms made by other members of the Royal Greenwich Observatory. 